\title{
Obesity and menopause modify the epigenomic profile of breast cancer
}

\author{
Ana B Crujeiras1,2,3, Angel Diaz-Lagares1,4, Olafur A Stefansson1,5, \\ Manuel Macias-Gonzalez ${ }^{3,6}$, Juan Sandoval1, Juan Cueva4, Rafael Lopez-Lopez4, \\ Sebastian Moran', Jon G Jonasson7, Laufey Tryggvadottir7, Elinborg Olafsdottir7, \\ Francisco J Tinahones ${ }^{3,6}$, Marcos C Carreira2,3, Felipe F Casanueva2,3,* and \\ Manel Esteller1,8,* \\ ${ }^{1}$ Cancer Epigenetics and Biology Program (PEBC), Bellvitge Biomedical Research Institute (IDIBELL), \\ Barcelona, Catalonia, Spain \\ 2Laboratory of Molecular and Cellular Endocrinology, Instituto de Investigación Sanitaria (IDIS), \\ Complejo Hospitalario Universitario de Santiago (CHUS/SERGAS), Santiago de Compostela University \\ (USC), Santiago de Compostela, Spain \\ ${ }^{3}$ CIBER Fisiopatología de la Obesidad y Nutrición (CIBERobn), Madrid, Spain \\ 4Translational Medical Oncology Group (Oncomet), Instituto de Investigación Sanitaria (IDIS); \\ Complejo Hospitalario Universitario de Santiago de Compostela (CHUS/SERGAS) and CIBER de \\ Cancer (CIBERONC), Santiago de Compostela, Spain \\ ${ }^{5}$ Cancer Research Laboratory, Faculty of Medicine, University of Iceland, Reykjavic, Iceland \\ 6Unidad de Gestión Clínica de Endocrinología y Nutrición, Instituto de Investigación Biomédica de \\ Málaga (IBIMA), Complejo Hospitalario de Málaga (Virgen de la Victoria), Universidad de Málaga, \\ Málaga, Spain \\ 7Department of Pathology and the Icelandic Cancer Registry, Icelandic Cancer society and Landspitali \\ University Hospital, Reykjavik, Iceland \\ ${ }^{8}$ Department of Physiological Sciences II, School of Medicine, University of Barcelona and Instituto \\ Catalana de Recerca i Estudis Avançats (ICREA), Barcelona, Catalonia, Spain \\ *(F F Casanueva and M Esteller contributed equally to this work)
}

Correspondence should be addressed to A B Crujeiras or F F Casanueva or M Esteller Email anabelencrujeiras@hotmail. com or endocrine@usc.es or mesteller@idibell.cat

\begin{abstract}
Obesity is a high risk factor for breast cancer. This relationship could be marked by a specific methylome. The current work was aimed to explore the impact of obesity and menopausal status on variation in breast cancer methylomes. Data from Infinium 450K array-based methylomes of 64 breast tumors were coupled with information on BMI and menopausal status. Additionally, DNA methylation results were validated in 18 non-tumor and 81 tumor breast samples. Breast tumors arising in either pre- or postmenopausal women stratified by $\mathrm{BMI}$ or menopausal status alone were not associated with a specific DNA methylation pattern. Intriguingly, the DNA methylation pattern identified in association with the high-risk group (postmenopausal women with high BMI (>25) and premenopausal women with normal or low $\mathrm{BMI}<25$ ) exclusively characterized by hypermethylation of $1287 \mathrm{CpG}$ sites as compared with the low-risk group. These $\mathrm{CpG}$ sites included the promoter region of fourteen proteincoding genes of which CpG methylation over the ZNF577 promoter region represents the top scoring associated event. In an independent cohort, the ZNF577 promoter methylation remained statistically significant in association with the high-risk group. Additionally, the impact of ZNF577 promoter methylation on mRNA expression levels was demonstrated in breast cancer cell lines after treatment with a demethylating agent (5-azacytidine). In conclusion, the epigenome of breast tumors is affected by a complex interaction between $\mathrm{BMI}$ and menopausal status. The ZNF577 methylation quantification is clearly relevant for the development of novel biomarkers of precision therapy in breast cancer.
\end{abstract}

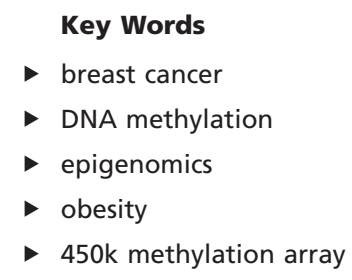

http://erc.endocrinology-journals.org DOI: $10.1530 / E R C-16-0565$
๑) 2017 Society for Endocrinology Printed in Great Britain
Published by Bioscientifica Ltd.
Endocrine-Related Cancer (2017) 24, 351-363 


\section{Introduction}

The prevalence of obesity and its associated co-disorders is rapidly increasing worldwide. Nowadays, these diseases are considered to be pandemic non-communicable diseases and are a major challenge for the health care system (Seidell \& Halberstadt 2015). Breast cancer, the leading cause of cancer diagnosis in women, is among the co-disorders associated with obesity. Overweight or obesity is a known risk factor for breast cancer (Lorincz \& Sukumar 2006), especially in postmenopausal females (Crujeiras et al. 2011, van Gemert et al. 2015). In contrast, being overweight or obese has no effect, or even a small protective effect, in premenopausal women. For every 5-unit increase in body mass index (BMI), the risk of developing postmenopausal breast cancer increases by $12 \%$, and elevated BMI is associated with a protective effect against the development of breast cancer in premenopausal women (Renehan et al. 2008). Notably, despite epidemiological and clinical evidence, the mechanisms that underlie the association between obesity and breast cancer risk are not completely understood. Obesity-induced inflammation promoted by adipose tissue dysfunction was proposed as an important link between obesity and cancer (Crujeiras et al. 2013, Howe et al. 2013).

Together with an imbalance in energy homeostasis, obesity is characterized by a state of chronic low-grade inflammation that promotes oxidative stress due to dysfunction in adipose tissue and alterations in adipocytederived hormone secretion and cytokine synthesis (Zou \& Shao 2008). Hormonal and inflammatory mediators can induce and maintain epigenetic regulation (Milagro et al. 2013, Yara et al. 2013). Frequent epigenetic modifications, such as DNA hypo- and hypermethylation and histone modifications, have been observed in several types of tumors, including breast cancer (Heyn et al. 2013, Stefansson \& Esteller 2013). This DNA methylation pattern is associated with an increased risk of cancer disease, a poor prognosis and a decreased likelihood of relapse-free survival (Hill et al. 2011). On the other hand, DNA methylation have also been found to be associated with obesity itself in a genome-wide DNA methylation analysis (Dick et al. 2014) and in analysis of specific genes (Milagro et al. 2012). Therefore, obesity could contribute to malignancies of the breast by altering the microenvironment such that it favors epigenetic changes and increases the susceptibility for inducing and maintaining a disease state (Crujeiras \& Casanueva 2015). Because epigenetic modifications are dynamic, reversible and change in response to dietary patterns, physical activity and weight loss, epigenetic markers related to obesity may represent therapeutic targets for the prevention of obesity-related disorders, including breast cancer. In this regard, previous studies have recently evaluated the associations between body mass and the methylation of a single gene or a panel of cancer-related genes in breast tumors (Tao et al. 2011, Naushad et al. 2014, Hair et al. 2015, McCullough et al. 2015). After these pioneer studies, examining the epigenome-wide association of breast cancer that depends on adiposity and the menopausal status could add novel insight to the epigenetic regulation of the obesity and breast cancer relationship.

The aim of the current work was to explore the effects of obesity and menopausal status on the DNA methylation profile of breast tumors using a genomewide DNA methylation approach and to identify an epigenetic signature of breast cancer taking into account the adiposity and menopausal state.

\section{Subjects and methods}

\section{Study participants}

A total of one hundred forty-five women diagnosed with histologically confirmed invasive breast cancer and eighteen healthy women were included in the current study (Table 1 and Fig. 1).

The discovery cohort was composed of breast tumor tissues (frozen tissues; $n=64$ ) obtained from the Department of Pathology, University Hospital, Iceland. Patient information associated with the breast cancer samples was obtained from the population-based Icelandic Cancer Registry. Informed consent was obtained from all patients, and all analyses were performed in accordance with the Icelandic Data Protection Commission (200650307) and Bioethics Committee (VSNb2006050001/03-16)

The validation cohort consisted of normal breast $(n=18)$ and breast cancer $(n=81)$ human paraffin-embedded (FFPE) tissue blocks from the BioBank Complejo Hospitalario Universitario de Santiago (CHUS) (PT13/0010/0068), integrated in the Spanish National Biobanks Network and they were processed following standard operating procedures with the appropriate approval of the Ethical and Scientific Committees (ref 2009/076).

Pre-diagnosis body weight, height, age and menopausal status were retrieved from medical records for all participants in the study. BMI was calculated as weight

Published by Bioscientifica Ltd 
Table 1 Clinical characteristics of the discovery and validation cohorts.

\begin{tabular}{|c|c|c|c|c|c|c|c|c|c|c|}
\hline & \multicolumn{4}{|c|}{$\begin{array}{c}\text { Discovery cohort } \\
\text { Tumor }\end{array}$} & \multicolumn{6}{|c|}{ Validation Cohort } \\
\hline & \multicolumn{4}{|c|}{ Tumor } & \multicolumn{4}{|c|}{ Tumor } & \multirow{2}{*}{\multicolumn{2}{|c|}{$\begin{array}{c}\text { Non-tumor } \\
\text { Obese }(n=18)\end{array}$}} \\
\hline & \multicolumn{2}{|c|}{ Normal-weight $(n=25)$} & \multicolumn{2}{|c|}{ Obese $(n=39)$} & \multicolumn{2}{|c|}{ Normal-weight $(n=27)$} & \multicolumn{2}{|c|}{ Obese $(n=54)$} & & \\
\hline & Mean & S.D. & Mean & S.D. & Mean & S.D. & Mean & S.D. & Mean & S.D. \\
\hline Age at diagnosis (years) & 49.5 & 9.8 & 53.2 & 10.9 & 51.9 & 13.2 & 61.2 & 12.4 & 44.9 & 16.8 \\
\hline Body weight $(\mathrm{kg})$ & 62.1 & 6.8 & 79.2 & 12.1 & 56.7 & 6.9 & 70.5 & 12.4 & 79.1 & 10.2 \\
\hline Height (m) & 1.64 & 0.06 & 1.65 & 0.07 & 1.58 & 0.06 & 1.56 & 0.59 & 1.61 & 0.06 \\
\hline BMI $\left(\mathrm{kg} / \mathrm{m}^{2}\right)$ & 22.9 & 1.6 & 29.1 & 4.0 & 22.7 & 2.2 & 30.4 & 5.0 & 30.6 & 4.2 \\
\hline Menopausal status (Pre/post) & \multicolumn{2}{|c|}{$16 / 9$} & \multicolumn{2}{|c|}{$15 / 24$} & \multicolumn{2}{|c|}{$17 / 10$} & \multicolumn{2}{|c|}{$13 / 41$} & \multicolumn{2}{|c|}{$11 / 7$} \\
\hline
\end{tabular}

in $\mathrm{kg}$ divided by the squared height in meters and was further categorized using the World Health Organization (WHO) criteria: normal/under-weight, $\mathrm{BMI}<25 \mathrm{~kg} / \mathrm{m}^{2}$; overweight, $25 \leq \mathrm{BMI}<30 \mathrm{~kg} / \mathrm{m}^{2}$ and obese, $\mathrm{BMI} \geq 30 \mathrm{~kg} / \mathrm{m}^{2}$

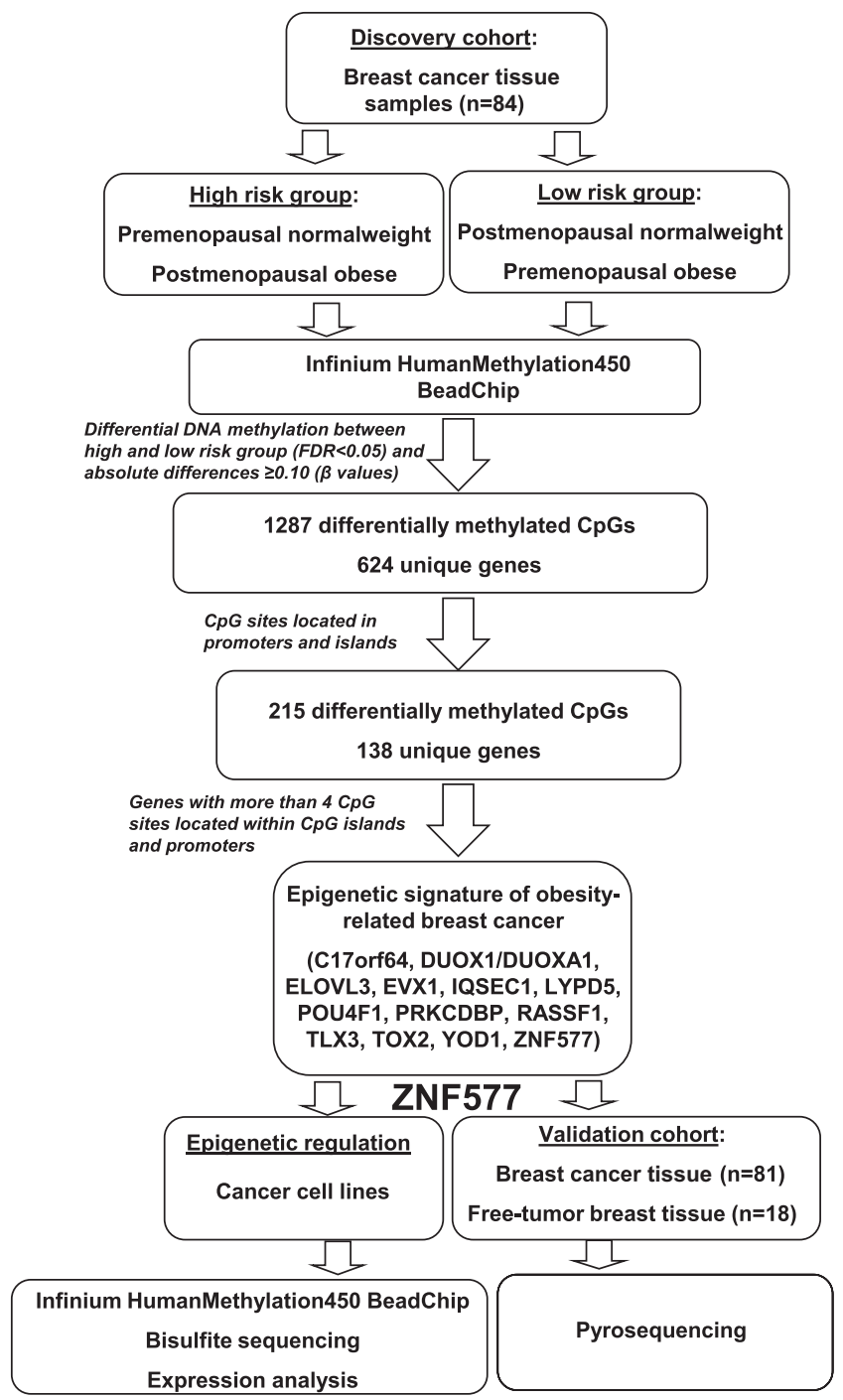

Figure 1

Schematic flow chart of DNA methylation and functional analysis.
(WHO, 2000). Then, patients were classified considering overweight and obese patients in the same group, as obese $\left(\mathrm{BMI}>25 \mathrm{~kg} / \mathrm{m}^{2}\right)$ and non-obese $\left(\mathrm{BMI} \leq 25 \mathrm{~kg} / \mathrm{m}^{2}\right)$ to evaluate the effect of excess body weight.

\section{DNA preparation and bisulfite conversion}

DNA from fresh-frozen (FF) tissue samples was isolated using a standard phenol-chloroform/proteinase-k protocol, whereas paraffin samples (FFPE) (10 sections of $14 \mu \mathrm{m}$ ) were processed using the E.Z.N.A. FFPE DNA kit (Omega Bio-Tek, Norcross, GA, USA), with a xylene wash to remove the paraffin. For each sample of tumor tissue, subsequent sections were stained with hematoxylin and eosin for histologic confirmation of the presence $(>50 \%)$ of tumor cells. The obtained DNA was treated with RNase A for $1 \mathrm{~h}$ at $45^{\circ} \mathrm{C}$. All DNA samples were quantified using the fluorometric method (Quan-iT PicoGreen DsDNA Assay, Life Technologies) and were assessed for purity using a NanoDrop (Thermo Scientific) with 260/280 and 260/230 ratio measurements. The integrity of the FF DNA was verified by electrophoresis in a $1.3 \%$ agarose gel. FF DNA (600 ng) and FFPE DNA (300 ng) were processed using the EZ-96 DNA Methylation kit (Zymo Research Corp, Irvine, CA, USA) following the manufacturer's recommendations for Infinium assays.

\section{DNA methylation analysis}

Infinium Human Methylation 450 BeadChip array Microarray-based DNA methylation analysis was conducted with the Infinium Human Methylation 450 BeadChip (450k array). DNA quality checks, bisulfite modification, hybridization, data normalization, statistical filtering and $\beta$ value calculations were performed as described elsewhere (Sandoval et al. 2011). High-quality DNA samples obtained from breast tumors (discovery cohort, $n=64$ ) were selected for bisulfite conversion (Zymo Research; EZ-96 DNA Methylation Kit) and

Published by Bioscientifica Ltd 
hybridization to Infinium Human Methylation 450 BeadChips (Illumina) following Illumina's Infinium HD methylation protocol. The DNA concentration of the quality control sample standards was measured using the PicoGreen method (Invitrogen), coupled with assessments of DNA purity based on the A260/A280 ratio (ranging between 1.75 and 1.95) and the A260/A230 ratio (ranging between 2.00 and 2.20). DNA fragmentation or RNA contamination was analyzed by $1 \%$ agarose gel electrophoresis. The Infinium Human Methylation 450 BeadChip provides coverage of $>450,000$ CpG sites targeting nearly all RefSeq genes (>99\%) (Sandoval et al. 2011). The chips were designed to cover coding and noncoding genes without bias against those lacking CpG islands. The design further aimed to cover not only promoter regulatory regions but also CpGs across gene regions to include $5^{\prime}$-untranslated regions (5' UTRs), the first exons, gene bodies and $3^{\prime}$-untranslated regions (3'-UTRs).

A total of $600 \mathrm{ng}$ from high-quality DNA samples was bisulfite converted. Whole-genome amplification and hybridization were then carried out on the BeadChip, followed by single-base extension and analysis on the HiScan SQ module (Illumina) to assess cytosine methylation states. The annotation of CGIs used the following categorization: (1) shore, for each of the $2-\mathrm{kb}$ sequences flanking a CGI; (2) shelf, for each of the 2-kb sequences next to a shore; and (3) open sea, for DNA not included in any of the previous sequences or in CGIs (Heyn et al. 2012). Transcription start site 200 or transcription start site 1500 indicates the region either 200 or $1500 \mathrm{bp}$ from the transcription start site, respectively.

Pyrosequencing analysis Pyrosequencing was used to assess selected markers in the validation cohort $(n=98$ FFPE breast tumors and $n=19$ normal FFPE mammary glands) as previously described (Heyn et al. 2012). The primer sequences used in this analysis were designed using Qiagen's PyroMark Assay Design 2.0 software to hybridize to CpG-free sites to ensure methylationindependent amplification (details and primer sequences are available in the Supplementary Table 1, see section on supplementary data given at the end of this article). Briefly, PCR was performed using standard conditions with biotinylated primers, and the PyroMark Vacuum Prep Tool (Biotage, Uppsala, Sweden) was used to prepare singlestranded PCR products according to the manufacturer's instructions. Pyrosequencing reactions and methylation quantification were performed in PyroMark Q96 System, version 2.0.6 (Qiagen) using appropriate reagents and recommended protocols.

Bisulfite genomic sequencing Methyl Primer Express v1.0 software was used to identify CpG islands and to design specific primers for the ZNF577 methylation analysis (Supplementary Table 1). DNA methylation status was determined using bisulfite genomic sequencing of multiple clones in DNA samples that were previously treated with sodium bisulfite (EZ DNA Methylation Gold Kit, Zymo Research).

\section{Cell lines and treatments for functional analysis}

Representative human breast cancer cell lines (MCF7, Hs578T, MDA-MB-468 and MDA-MB-134IV) and normal epithelial breast cell line (MCF10A) from American Type Culture Collection (Manassas, VA) were used to evaluate the DNA methylation and gene expression of ZNF577. Cells were cultured in DMEM containing 10\% FBS, penicillin and streptomycin at $37^{\circ} \mathrm{C}$ and $5 \% \mathrm{CO}_{2}$. To restore the expression of DNA-methylated ZNF577, the MCF7 cells were treated with the DNA demethylating agent 5-aza-2'-deoxycytidine (A3656, Sigma) at $5 \mu \mathrm{M}$ for $72 \mathrm{~h}$. All the cell lines were obtained from the American Type Culture Collection (ATCC; Manassas, VA) and were used with passage lower than 15. ATCC authenticates the cell lines routinely following a very strict test that includes short tandem repeat (STR) profiling, karyotyping and cytochrome $\mathrm{C}$ oxidase I (COI) testing.

\section{Expression assays by qRT-PCR and RT-PCR}

RNA from the cell culture assays was extracted using the Thermo Scientific GeneJet RNA Purification Kit (Thermo Scientific, K0739). The RNA concentrations were measured with a NanoDrop 2000 spectrophotometer (Thermo Scientific). cDNA was synthesized using the High-Capacity cDNA Reverse Transcription Kit (Applied Biosystems) with $2 \mu \mathrm{g}$ of total RNA. qRT-PCR was performed using TaqMan Universal PCR Master Mix, TaqMan Probes (Applied Biosystems) and the Step OnePlus Real-Time PCR System (Applied Biosystems). All experiments were performed in duplicate, and gene expression levels were normalized to those of the housekeeping genes ACTB or GAPDH, depending on the sample. The fold change in gene expression was calculated using the $2^{-\Delta \Delta C t}$ relative quantitation method according to the manufacturer's guidelines 
(Applied Biosystems), and data are reported as the geometric mean (s.E.M.).

\section{Statistical analyses}

The methylation level of each cytosine was expressed as a $\beta$ value, which was calculated as the fluorescence intensity ratio of the methylated to the unmethylated versions of the probes. $\beta$ values ranged between 0 (unmethylated) and 1 (completely methylated) according to a combination of the Cy3 and Cy5 fluorescence intensities. Color balance adjustment and quantile normalization were performed to normalize the samples between the two color channels. $\beta$ values with a detection $P$ value $>0.01$ are considered to fall below the minimum intensity and threshold, and these values were consequently removed from further analysis. Approximately 96\% of CpG islands were covered, along with regions proximal to $\mathrm{CpG}$ islands ('CpG shores') and the more distal CpG shelves. Additionally, probes that were localized to the sex chromosomes and those considered to be single nucleotide polymorphisms (SNPs) were filtered out.

Samples were classified in the discovery and validation cohorts according to adiposity and menopausal status. The genome-wide DNA methylation patterns were first compared for tumors from obese and normal-weight patients. Then, the samples were categorized into two risk groups that combined adiposity and menopausal status, based on the epidemiological data that reported a higher risk of breast cancer in obese postmenopausal women compared to obese premenopausal women (Crujeiras et al. 2011). The high-risk group was composed of samples from obese postmenopausal and normalweight premenopausal women, and the low-risk group was composed of obese premenopausal and normalweight postmenopausal women.

To identify consistent patterns of differentially methylated CpG sites between obese and normal-weight samples and between the high-risk group and group $\mathrm{B}$, we carried out a univariate permutation-based approach using the SAMr package for R. By this analysis, false discovery rates (FDR) below 5\% were considered statistically significant. In addition, we applied a threshold for the significant sites using mean differences between groups, with a minimum change of \pm 0.10 in $\beta$ values. Hierarchical cluster analysis of the significant CpGs was performed using the heatmap function in $\mathrm{R}$.

To estimate the enrichment in biological processes, a hypergeometric test was performed on biological processes defined by gene ontology (GO) (Falcon \& Gentleman 2007). This analysis detects significant over-representation of GO terms in one of the sets (list of selected genes) with respect to the other for the entire genome. GO terms with an adjusted $P$ value $<0.05$ were considered significant.

Additionally, the diagnostic efficiency (percent of correctly classified) of the candidate genes differentially methylated was calculated as the percent of agreement by using the receiver-operating characteristic (ROC) curve analysis. These results are often interpreted as negligible efficiency $(<20 \%)$, minimal (20-40\%), moderate (41-60\%), good (61-80\%) and excellent (>80\%).

\section{Results}

\section{Characteristics of patients}

Among the 64 patients with primary breast cancer included in the discovery cohort, $60.9 \%$ were classified as overweight/obese, $39.1 \%$ were normal weight and $51.6 \%$ were postmenopausal. In the validation cohort, $71.4 \%$ patients were classified as overweight/obese, 28.6\% were normal weight and $61.2 \%$ were postmenopausal. The clinical characteristics of the discovery and validation cohorts are shown in Table 1.

\section{DNA methylation analysis by adiposity and menopausal state}

DNA methylation profiling in human breast tumors involving the analysis of approximately 450,000 CpGs were analyzed with respect to BMI and menopausal status. Firstly, tumors arising in obese or overweight (BMI >25) women were compared with those arising in normal weight women (BMI <25). This analysis revealed no statistically significant differences following adjustment for multiple hypothesis testing. Secondly, no significant differences were detected between tumors arising in premenopausal women when compared with tumors arising in postmenopausal women.

Epidemiological studies have consistently identified high BMI (>25) in postmenopausal women as a risk factor for developing breast cancer (Crujeiras et al. 2011). This observation gives reason to specifically determine whether the DNA methylation profiles of tumors arising in these highest-risk women (BMI $>25$ and postmenopausal) differ from the breast tumors from postmenopausal normalweight (BMI <25) women in the cohort. However, this analysis did not reveal any significantly associated CpGs after adjustment for multiple hypothesis testing.

Published by Bioscientifica Ltd. 
Another high-risk group defined on the basis of BMI and menopausal status includes premenopausal women with normal or low BMI (BMI <25). Similarly, tumors arising in this risk group, i.e. in premenopausal women with normal or low BMI, did not differ significantly with respect to normal-weight postmenopausal women. However, when combining together tumors from these two high-risk groups, i.e. postmenopausal women having BMI > 25 together with premenopausal women having BMI $<25$, we detected statistically significant differences involving 1,287 CpGs with FDR $<5 \%$ corresponding to 624 unique genes (see detailed list in Supplementary Table 2). The differentially methylated CpGs are exclusively characterized as changes towards CpG hypermethylation occurring during tumor development in the high-risk group (Fig. 2A). Notably, these differentially methylated CpG sites between the high- and low-risk groups were mainly located in promoters and $\mathrm{CpG}$ islands (Fig. 2B), suggesting the existence of a different methylator phenotype between both risk groups.

The CpG sites representing promoter regions (TSS1500, TSS200, 5' UTR and 1 $1^{\text {st }}$ exon) at CpG islands were selected to investigate biological relevance. This selection yielded 215 CpGs representing 138 unique genes (Supplementary
Table 3). The CpGs with the greatest change in DNA methylation $(>15 \%)$ are listed as representatives of the 215 CpG sites in Table 2.

Additionally, a gene ontology (GO) analysis was performed to determine whether some molecular functions or biological processes were significantly associated with these differentially methylated genes represented by CpGs located in promoter regions and CpG islands. Thus, among the significantly enriched biological processes, the top overrepresented categories were related to the regulation of transcription, signal transduction, the regulation of cell differentiation and proliferation, cell migration and cell cycle regulation (Fig. $2 \mathrm{C})$. Most of the genes (76\%) regulated by methylation belonged to a network significantly enriched in protein interactions $(P<0.001)$, according to STRING analysis (Supplementary Fig. 1).

To identify potentially novel signatures of DNA methylation in the high-risk group, those genes with more than $4 \mathrm{CpG}$ sites located within $\mathrm{CpG}$ islands and promoters and a difference in $\beta$-values $\geq 10 \%$ were selected (Supplementary Table 3). With these criteria, C17orf64, DUOX1/DUOXA1, ELOVL3, EVX1, POU4F1, TLX3, TOX2 and YOD1 were represented by 4 sites; IQSEC1 and

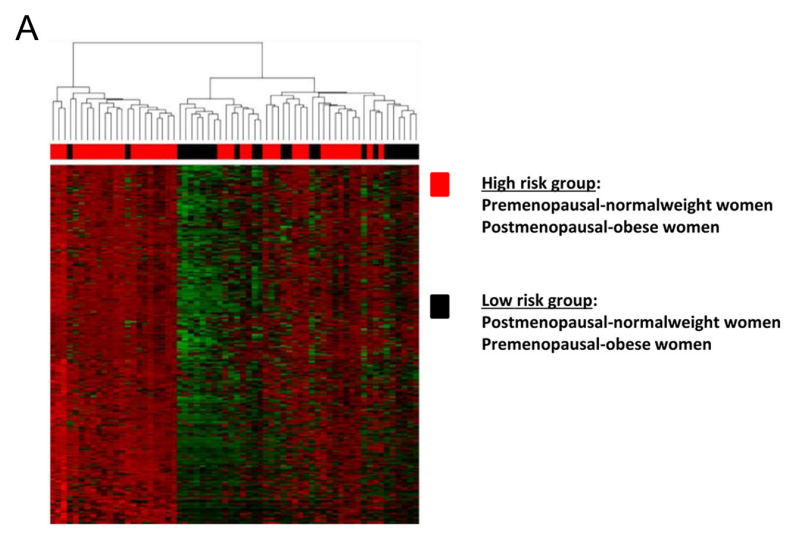

B

CpG content
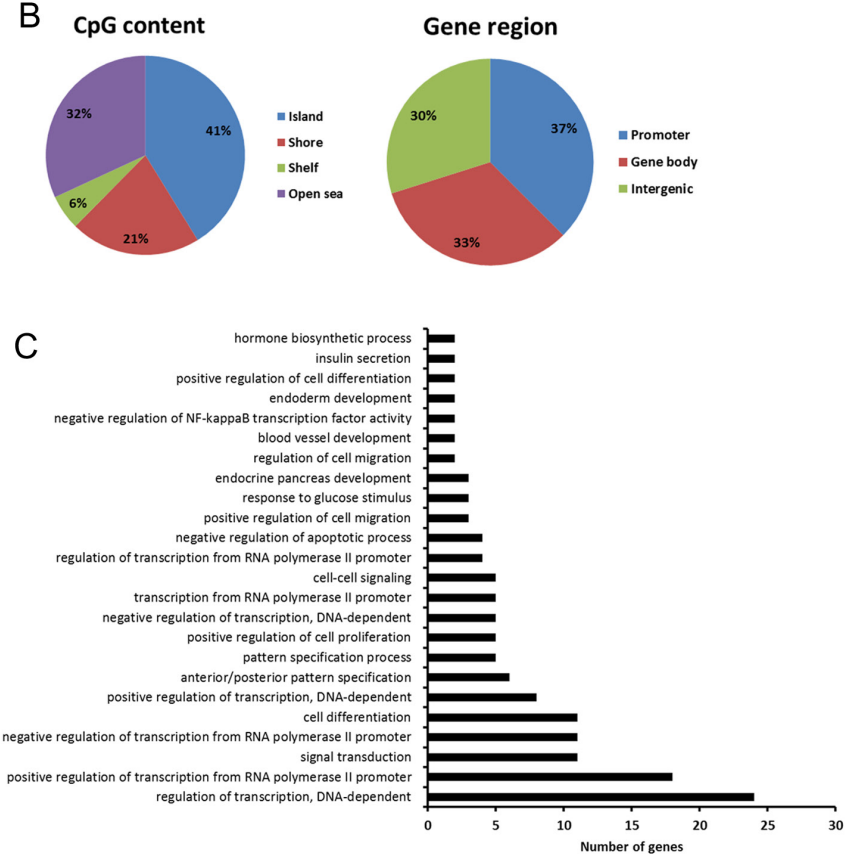

Figure 2

Characteristics of the candidate differentially methylated CpGs. (A) Supervised clustering of the $1287 \mathrm{CpGs}$ found differentially methylated between the high-risk and low-risk groups identified by $450 \mathrm{k}$ array analysis. (B) Genomic distribution of the differentially methylated CpG sites and their respective location regarding the broader CpG context and the gene region. Data represent DMCpGS in a region divided by the total DMCpGS (1282). (C) Summary of the gene ontology (GO) analysis of the biological process categories for differentially methylated genes represented by $\mathrm{CpG}$ sites located in promoter regions and islands. 
Table 2 The forty-two highest differentially methylated CpGs between high- and low-risk groups in promoter and island. Bold denotes genes with more than 4 differentially methylated CpG sites located within CpG islands and promoters and a difference in $\beta$-values $\geq 10 \%$.

\begin{tabular}{|c|c|c|c|c|c|c|c|}
\hline TargetID & Chr & Position & Gene name & Gene region & $\begin{array}{l}\text { CpG } \\
\text { Island }\end{array}$ & $\begin{array}{c}\text { Mean } \\
\text { differences } \\
\text { (Low-High) }\end{array}$ & $\begin{array}{c}\text { P value } \\
(t \text { test })\end{array}$ \\
\hline $\operatorname{cg} 10635122$ & 19 & 52391090 & ZNF577;ZNF577;ZNF577;ZNF577 & Body;5'UTR;5'UTR;1stExon & $\overline{\text { Island }}$ & -0.1570 & 0.0007 \\
\hline cg03562414 & 19 & 52391078 & ZNF577;ZNF577;ZNF577 & Body;5'UTR;5'UTR & Island & -0.1615 & 0.0007 \\
\hline cg20361154 & 22 & 22862884 & ZNF280B & 5'UTR & Island & -0.1615 & 0.0005 \\
\hline cg12042659 & 19 & 58951599 & ZNF132 & TSS200 & Island & -0.1670 & 0.0024 \\
\hline cg03206401 & 2 & 130956119 & TUBA3E & TSS200 & Island & -0.1527 & 0.0089 \\
\hline cg06779449 & 20 & 42544663 & TOX2;TOX2;TOX2 & TSS200;Body;TSS200 & Island & -0.1594 & 0.0011 \\
\hline cg06962944 & 20 & 42544728 & TOX2;TOX2;TOX2 & TSS200;Body;TSS200 & Island & -0.1518 & 0.0033 \\
\hline cg27089768 & 14 & 96180598 & TCL1A;TCL1A & TSS200;TSS200 & Island & -0.1581 & 0.0018 \\
\hline cg04550737 & 1 & 119530600 & TBX15 & 5'UTR & Island & -0.1797 & 0.0010 \\
\hline cg13996209 & 9 & 33447594 & SUGT1P1;AQP3 & Body;TSS200 & Island & -0.1710 & 0.0007 \\
\hline cg01934626 & 5 & 180018562 & SCGB3A1 & TSS200 & Island & -0.1805 & 0.0040 \\
\hline cg22859061 & 5 & 180018503 & SCGB3A1 & TSS200 & Island & -0.1727 & 0.0040 \\
\hline cg27569446 & 3 & 50378431 & RASSF1;RASSF1 & TSS200;TSS200 & Island & -0.1530 & 0.0066 \\
\hline cg26678920 & 11 & 6341888 & PRKCDBP & TSS200 & Island & -0.1748 & 0.0001 \\
\hline cg09010671 & 13 & 79177763 & POU4F1 & TSS200 & Island & -0.1511 & 0.0040 \\
\hline cg22287064 & 17 & 73584070 & MYO15B & TSS200 & Island & -0.1856 & 0.0009 \\
\hline cg00397324 & 21 & 42798594 & $M \times 1 ; M \times 1$ & 5'UTR;5'UTR & Island & -0.1503 & 0.0008 \\
\hline cg09734791 & 8 & 72756155 & MSC & 1stExon & Island & -0.1864 & 0.0005 \\
\hline cg14409559 & 8 & 72756341 & MSC & 1stExon & Island & -0.1699 & 0.0006 \\
\hline cg02523844 & 7 & 79083507 & MAGI2 & TSS1500 & Island & -0.1515 & 0.0016 \\
\hline cg16896847 & 8 & 144512042 & MAFA & 1stExon & Island & -0.1806 & 0.0008 \\
\hline cg25732462 & 19 & 44324898 & LYPD5 & TSS200 & Island & -0.1608 & 0.0003 \\
\hline cg18467168 & 3 & 13114803 & IQSEC1 & TSS200 & Island & -0.1651 & 0.0003 \\
\hline cg27246282 & 3 & 13114797 & IQSEC1 & TSS200 & Island & -0.1555 & 0.0004 \\
\hline cg14335894 & 17 & 47075281 & IGF2BP1;IGF2BP1 & 1stExon;1stExon & Island & -0.1500 & 0.0036 \\
\hline cg08260959 & 6 & 26240920 & HIST1H4F & 1stExon & Island & -0.1534 & 0.0011 \\
\hline cg02578368 & 6 & 26250744 & HIST1H2BH;HIST1H3F & TSS1500;1stExon & Island & -0.1540 & 0.0006 \\
\hline cg15975865 & 2 & 127413831 & GYPC;GYPC;GYPC;GYPC & 5'UTR;1stExon;5'UTR;1stExon & Island & -0.1574 & 0.0051 \\
\hline cg00582971 & 5 & 178422128 & GRM6 & TSS200 & Island & -0.1509 & 0.0032 \\
\hline cg05621343 & 11 & 134146253 & GLB1L3 & TSS200 & Island & -0.1796 & 0.0004 \\
\hline cg16087093 & 5 & 169533347 & FOXI1;FOXI1 & 1stExon;1stExon & Island & -0.1515 & 0.0031 \\
\hline cg15841063 & 1 & 63789500 & FOXD3 & 1stExon & Island & -0.1699 & 0.0007 \\
\hline cg24402300 & 19 & 55591437 & EPS8L1;EPS8L1 & TSS1500;Body & Island & -0.1678 & 0.0006 \\
\hline cg13447927 & 10 & 103986128 & ELOVL3 & TSS200 & Island & -0.1581 & 0.0001 \\
\hline cg08328777 & 15 & 45422004 & DUOX1;DUOX1;DUOXA1;DUOXA1 & TSS200;TSS200;1stExon;5'UTR & Island & -0.1657 & 0.0009 \\
\hline cg05804220 & 15 & 45422062 & DUOX1;DUOX1;DUOXA1 & TSS200;TSS200;TSS200 & Island & -0.1550 & 0.0019 \\
\hline cg05377226 & 1 & 171810910 & DNM3;DNM3 & 1stExon;1stExon & Island & -0.1752 & 0.0020 \\
\hline cg04887807 & 4 & 5021311 & CYTL1 & TSS200 & Island & -0.1792 & 0.0004 \\
\hline cg03199564 & 4 & 5021084 & CYTL1 & 1stExon & Island & -0.1590 & 0.0054 \\
\hline cg27247697 & 2 & 87018054 & $\mathrm{CD} 8 \mathrm{~A} ; \mathrm{CD} 8 \mathrm{~A} ; \mathrm{CD} 8 \mathrm{~A} ; \mathrm{CD} 8 \mathrm{~A} ; \mathrm{CD} 8 \mathrm{~A} ; \mathrm{CD} 8 \mathrm{~A}$ & $\begin{array}{l}\text { 5'UTR;1stExon;5'UTR;Body; } \\
\text { 5'UTR;1stExon }\end{array}$ & Island & -0.1552 & 0.0004 \\
\hline cg09695735 & 17 & 58498977 & C17orf64 & TSS1500 & Island & -0.2482 & 0.0001 \\
\hline cg10179315 & 17 & 58498871 & C17orf64 & TSS1500 & Island & -0.1972 & 0.0001 \\
\hline
\end{tabular}

LYPD5 were represented by 5 sites, and PRKCDBP and RASSF1, by 6 sites. Relevantly, ZNF577 was the mostrepresented gene, with 7 CpGs that were differentially methylated in promoter region and island with high statistical significance.

\section{Validation of candidate genes in an independent cohort}

We used pyrosequencing, a technique that is most feasible for studies of patients in hospitals, to evaluate the DNA methylation levels for RASSF1 and ZNF577 in an independent cohort of paraffin samples from breast cancer patients and cancer-free donors (validation cohort; $N=81$ breast tumors and $N=18$ non-tumoral breast tissues; Table 1).

We confirmed significant higher levels of methylation for RASSF1 in tumor samples compared with non-tumoral donors (Supplementary Fig. 2A). When the methylation levels were analyzed in breast tumors according to the 
adiposity and menopausal state, no differences were observed in RASSF1 (Supplementary Fig. 2B and C). Regarding ZNF577, the epigenetic regulation in breast tumors has not been previously described. Therefore, first the methylation levels were evaluated by comparing tumor with non-tumoral samples. A significant increase in methylation levels was observed for ZNF577 in tumor compared with non-tumoral samples in both, our cohort (Fig. 3A) and an independent cohort from The Cancer Genome Atlas; TCGA (Supplementary Fig. 3). When the methylation levels were analyzed in breast tumors according to adiposity and menopausal state, ZNF577 exhibited higher methylation levels in postmenopausal obese and premenopausal normal-weight women than in the other cohort groups (Fig. 3B). In fact, the statistical differences observed in the Infinium Human Methylation 450 BeadChip array between the high-risk group and lowrisk group were corroborated by pyrosequencing, with statistical significance in ZNF577 methylation levels (Fig. 3C). Notably, the methylation levels of the promoter region of ZNF577 (Fig. 4A) assessed in different breast tumor cell lines by bisulfite genomic sequencing analysis (Fig. 4B) and by the Infinium Human Methylation 450 BeadChip array (Fig. 4C) were inversely correlated with the transcript levels of ZNF577 (Fig. 4D). Moreover, after treating breast cancer cell lines that exhibited ZNF577 hypermethylation with the demethylating agent 5-aza-2'-deoxycytidine, the expression of this gene was significantly restored (Fig. 4E), indicating that DNA methylation has a functional role in the transcriptional control of ZNF577.

Receiver-operating characteristic (ROC) curves were used to evaluate the ability of ZNF577 methylation levels to discriminate tumor from non-tumor as well as highrisk from low-risk patients. The area under the ROC curve (AUC) of ZNF577 methylation levels was $0.93(P<0.001)$ for breast tumor detection (Fig. 5A) and $0.69(P=0.008)$ to discriminate the high-risk from low-risk group (Fig. 5B).

\section{Discussion}

Epigenetic modifications of the genome mediate the response of the organism to environmental pressure. There is a growing body of evidence that demonstrates a relevant role for epigenetic marks in susceptibility

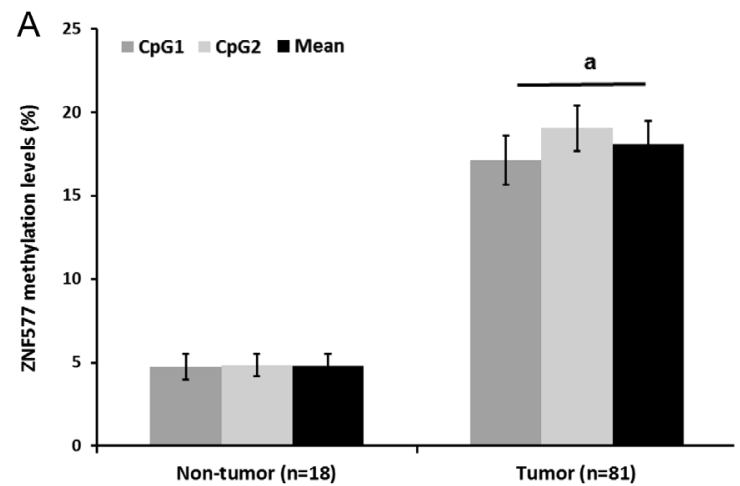

B

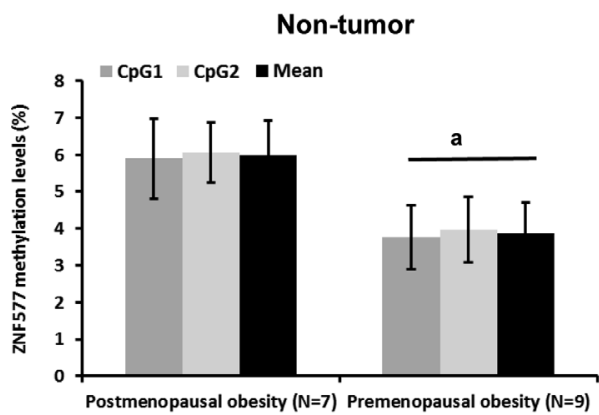

C

Tumor

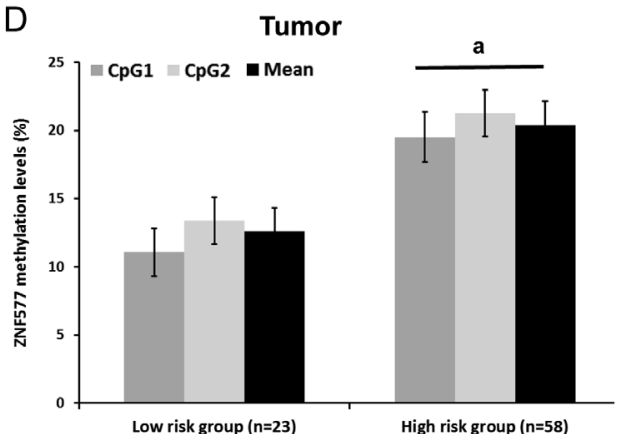

Figure 3

Methylation analysis of ZNF577 by pyrosequencing. (A) Methylation level differences between tumor and non-tumor breast tissues. (B) Methylation levels in non-tumor breast tissues from obese patients, depending on the menopausal state. (C) Methylation levels in breast tumor tissues, depending on obesity and menopausal state. (D) Methylation levels in breast tumor tissues in the high-risk and low-risk groups. Statistical analysis was evaluated using Student $t$ test and significance symbols correspond to a. $P<0.05$ vs non-tumor, premenopausal obesity or premenopausal normal-weight, respectively; $b$. $P<0.05$ vs premenopausal overweight/obesity; $C$. $P<0.05$ vs postmenopausal normal-weight. 
A

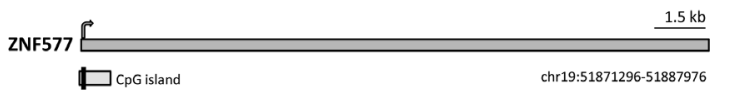

B

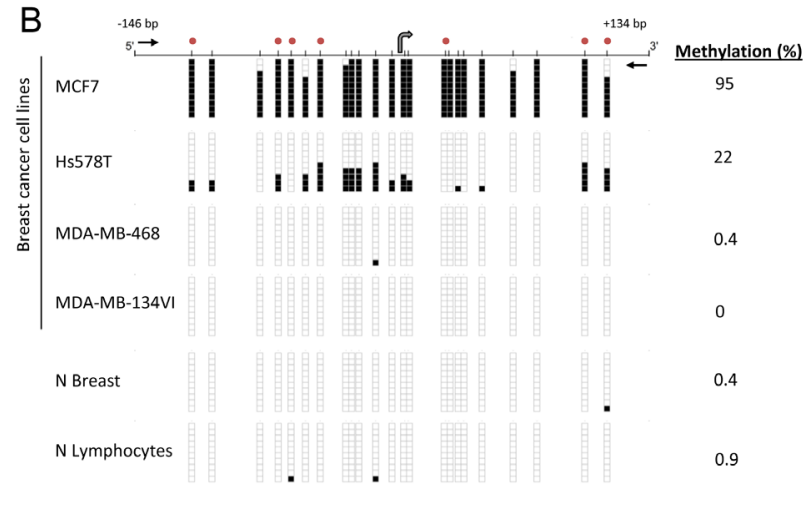

C

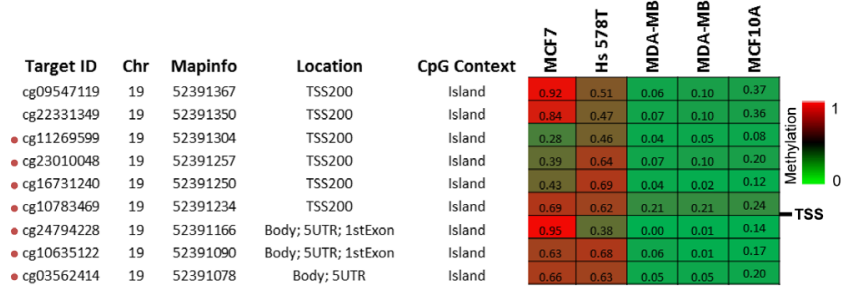

D

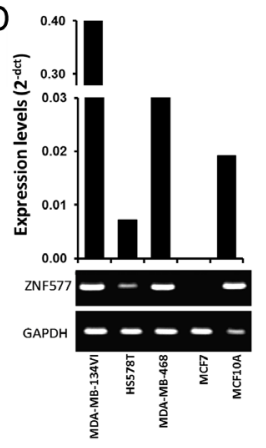

E

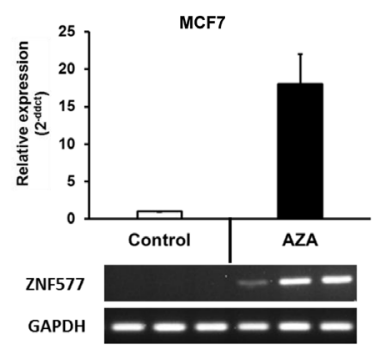

Figure 4

DNA methylation-associated silencing of ZNF577 breast cancer. (A) Schematic representation of ZNF577 genomic loci and CpG island. (B) Bisulfite genomic sequencing analysis of ZNF577 CpG island in human breast cancer cell lines and normal breast and lymphocytes as tissue controls. Location of bisulfite genomic sequencing PCR primers (black arrows), CpGs (vertical lines) and the transcriptional start site (grey arrow) are shown. Ten single clones are represented for each sample. Presence of unmethylated or methylated CpGs is indicated by white or black squares, respectively. Red circles represent the location of CpGs detected by the 450k methylation array. (C) ZNF577 methylation levels of breast cancer cell lines analyzed by $450 \mathrm{k}$ methylation array. (D) ZNF577 expression levels in methylated or unmethylated human breast cancer cell lines and in normal breast as control by RT-PCR and qRT-PCR Values were determined by qRT-PCR in triplicates and are expressed as mean $(n=3)$. (E) Restored ZNF577 expression after treatment with DNA demethylating agent 5-aza-2'-deoxycytidine (AZA) in ZNF577 CpG island methylated MCF7 cell line. Values were determined in triplicate by RT-PCT and qRT-PCR of three independent experiments and are expressed as mean \pm S.E.M $(n=3)$.

to obesity and its co-diseases. The results presented herein demonstrate that the impact of obesity on the breast cancer features could be mediated by a specific alteration of the methylation profile, particularly in cases of postmenopausal breast cancer. The epigenomewide analysis identified 138 genes that exhibited a differential methylation in the promoter region and island and they comprised an epigenetic signature of high-risk breast cancer by including obesity-related postmenopausal and normal weight premenopausal women. Among the identified signature genes, ZNF577 was the most relevant and its differential methylation levels were validated in an independent cohort. In addition, we demonstrate that ZNF577 gene expression is epigenetically regulated. The methylation levels of ZNF577 vary depending on the type of breast cancer cell line. This fact is in agreement with the heterogeneity of primary tumors of breast cancer, which is associated with clinical outcome of disease. Therefore, understanding the function of the methylation pattern of ZNF577 in response to therapy could be relevant to improving management of the disease taking into account adiposity and menopausal state.
To the best of our knowledge, the current study used the largest cohort to highlight the impact of obesity on primary breast tumor methylation profiles and to identify genome-wide high-resolution DNA methylation patterns that depend on menopausal status. Prior research has reported an association between obesity and methylation in tumor tissue from women diagnosed with breast cancer. These studies were focused on individual genes (Tao et al. 2011, Naushad et al. 2014) or on a panel of cancer-related genes (Hair et al. 2015, McCullough et al. 2015). For example, Hair and coworkers (Hair et al. 2015) demonstrated that obesity is associated with the degree of methylation in some cancer-relevant genes. Although the candidate genes that were differentially methylated in the former study were not validated in an independent cohort, and the functional relevance of the identified candidate genes was not evaluated, the results supposed new and relevant evidence on the epigenetic regulation of cancer-related genes induced by obesity in breast tumors. The current work adds new information to this issue by performing a comprehensive genome-wide analysis of the DNA methylation that interrogates 485,000 CpG sites. In the current work, we first compared the breast

Published by Bioscientifica Ltd 

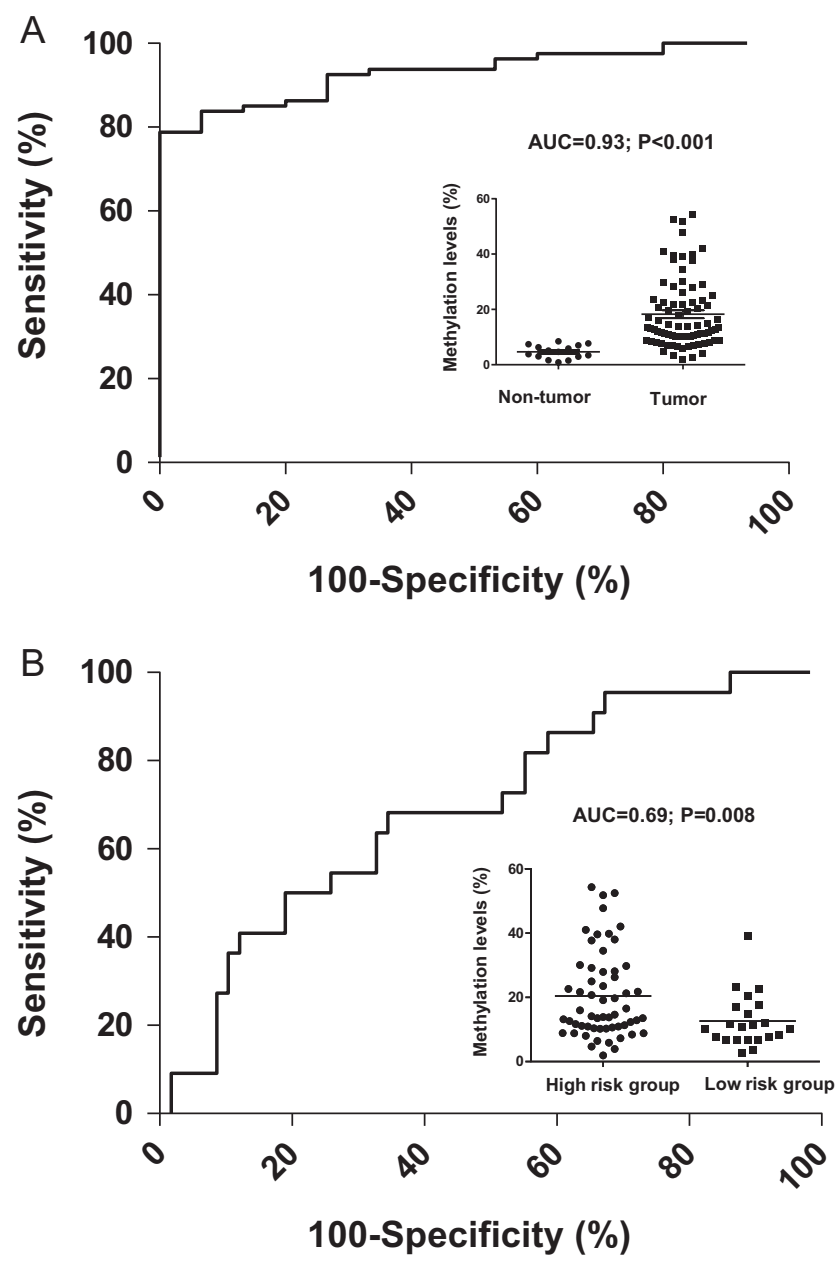

Figure 5

Receiver-operating characteristic (ROC) curves for ZNF577 methylation levels. Ability to discriminate tumor from non-tumor samples (A) and high-risk from low-risk groups (B).

tumors from obese vs normal-weight women, and we did not find statistically differences after adjusting for multiple comparisons. Then, we compared the obese with normal-weight samples grouping for premenopausal or postmenopausal state. Again, no differences were observed after adjusting for multiple comparisons. This lack of statistical significance could be due to the sample size yielded from the classification of the samples into 4 groups. Under this situation, the breast cancer patients were further classified into two groups according to their adiposity and menopausal status, considering that the effect of obesity on breast cancer is modulated by menopausal status (Crujeiras et al. 2011). In agreement with our working hypothesis, these two groups were differentiated by their DNA methylation profile. The CpGs that were differentially methylated were mainly hypermethylated in the high-risk group, and these CpGs were located in genes involved in several pathways related to carcinogenesis. Some genes previously reported to be differentially methylated in cancer were found among the highest differentially methylated CpGs in promoter regions and CpG islands. For example, among the genes represented by more than $4 \mathrm{CpGs}$, high methylation levels were observed for TOX2 (Tessema et al. 2012), RASSF1 (Conway et al. 2014), PRKCDBP (Li et al. 2015) and POU4F1 (Faryna et al. 2012). Other genes with increased methylation in the high-risk group were also previously reported as hypermethylated in cancer, such as IQSEC1 (Dmitriev et al. 2012). The dual NADPH oxidases were proposed as potential targets for cancer therapy because their overexpression in many tissues is associated with premalignant conditions (Roy et al. 2015). However, in the current work, increased methylation levels were found in CpGs associated with the dual oxidases DUOX/DUOXA1, similar to that observed in lung cancer (Luxen et al. 2008). On the other hand, genes associated with lipid metabolism and obesity features were observed such as ELOVL3 (Zadravec et al. 2010). Thus, the DNA methylation of the identified genes could represent an epigenetic signature that could be relevant in the personalized management of the breast cancer associated to obesity.

Additionally, we selected RASSF1 and ZNF577 for further validation in an independent cohort among the identified differentially methylated gene signatures of high-risk breast cancer depending on adiposity and menopausal state. RASSF1 a gene characteristically methylated in breast cancer (Conway et al. 2014) and was represented by $5 \mathrm{CpG}$ sites in the current work and ZNF577 was the most represented gene in the differential methylated profile of high-risk group with the highest statistical differences between the groups. According to previous work, RASSF1 exhibited high methylation levels in tumor breast than non-tumor breast tissue. However, the differential methylation pattern in tumor breast tissue according to adiposity and menopausal state was not validated in our independent cohort. Importantly, in this study, increased methylation of ZNF577 was demonstrated for the first time in breast tumors but not in non-malignant breast tissue. Moreover, the methylation profile of ZNF577 observed in obesityrelated postmenopausal breast tumors was observed in an independent cohort. Accordingly, the ZNF577 CpG island was previously found to be methylated in $85 \%$ to $100 \%$ of squamous cell carcinomas of the lung (Rauch et al. 2012), and in polycythemia vera, aberrant methylation of ZNF577 was correlated with differential expression of the ZNF577 gene (Barrio et al. 2011). Likewise, a previous

Published by Bioscientifica Ltd 
genome-wide association study found three SNPs from the ZNF577 gene region that were associated with breast cancer susceptibility (Sehrawat et al. 2011). In this line, an excellent power of efficiency for discriminating between the high- and low-risk groups of tumor samples was detected with the methylation levels of ZNF577 in the current work.

Zinc finger proteins regulate gene transcription. Although the functional role of ZNF577 is currently unknown, individual ZNFs, and even some clusters of ZNFs, are often hypermethylated and silenced in different types of tumors, suggesting that the current findings represent a commonly disrupted epigenetic pathway in cancer progression. For example, such a disrupted epigenetic mechanism was found in a model of arsenite-transformed prostate epithelia (Severson et al. 2013). Several studies have identified individual ZNFs as potential tumor suppressors that regulate cellular proliferation by inhibiting MAPK signaling and repressing various oncogenes (Severson et al. 2013). Previous research suggests that oxidative stress could be a possible mechanism by which ZNFs are hypermethylated during malignant transformation (Severson et al. 2013) because oxidative stress disrupts the CTCF/PARP-1 interaction, leading to increases in DNA methylation and other repressive epigenetic marks at corresponding genomic loci (Severson et al. 2013). Similarly, the impact of obesity on the epigenetic regulation of breast tumors could be mediated by oxidative stress (Crujeiras et al. 2013). However, additional studies are required to understand the functional role of ZNF577 in breast cancer biology as well as its utility in the personalized treatment of obese patients with breast cancer. In this line, the ZNF132, also identified in the current work as differentially methylated, was discovered among the epigenetic markers of an epi-subtype of the Luminal-B subtype of breast cancer (Stefansson et al. 2015). These results evidenced a potential role of the ZNFs for identifying tumor subtypes regardless of their specific function on carcinogenesis. Relevantly, the differential pattern of ZNF577 methylation levels found in cell lines from different breast cancer subtypes is in agreement with a potential role of ZNF577 in the heterogeneity of these tumors that could determine the response to anticancer therapies.

The strength and novelty of the current work are represented by the epigenome-wide analysis of a large discovery cohort that included data on BMI and menopausal state and the validation of the identified genes in an independent cohort. This study was performed in breast tumor samples; however, the use of FFPE tissues in the validation cohort, in contrast to FF tissues in the discovery cohort, could condition the validation of other epigenetic markers.

In conclusion, the current findings demonstrate the potential of methylation profiles to distinguish subtypes of breast cancer that depend on adiposity and menopausal state. The epigenome of breast tumors is affected by a complex interaction between BMI and menopausal status. Thus, patients with breast cancer associated with the described DNA methylation markers might also receive DNA demethylation agents as co-adjuvant therapy to the standard therapeutic protocol. Although further studies are needed, ZNF577 may be a biomarker of the obesity and menopausal state effect on breast cancer biology and a suitable therapeutic target in precision medicine.

\section{Supplementary data}

This is linked to the online version of the paper at http://dx.doi.org/10.1530/ ERC-16-0565.

\section{Declaration of interest}

The authors declare that there is no conflict of interest that could be perceived as prejudicing the impartiality of the research reported.

\section{Funding}

This work was supported by grants from the European Research Council (ERC) grant EPINORC; the Fondo de Investigacion Sanitaria (PI14/01012; PE13/00024) research project, and CIBERobn (CB06/003) from the Instituto de Salud Carlos III (ISCIII)-Subdireccion General de Evaluacion y Fomento de la Investigacion; Fondo Europeo de Desarrollo Regional (FEDER), Spain; the Health and Science Departments of the Catalan Government (Generalitat de Catalunya) and the Health Department of the Xunta the Galicia (GRC2014/034), Spain, as well as Fundación Lilly and Fundación Mapfre. Crujeiras A B was funded by the ISCIII through a research contract 'Sara Borrell' (C09/00365). Diaz-Lagares A is funded by the ISCIII through a research contract Río Hortega (CM14/00067). Macias-Gonzalez M is recipient of the Nicolas Monardes program from the Servicio Andaluz de Salud, Junta de Andalucia, Spain (Grant C-0029-2014). Sandoval J is a 'Miguel Servet' researcher. Esteller M is an ICREA Research Professor.

\section{Author's contribution statement}

The authors' responsibilities were as follows-A B C, F F C and M E: Project conception and leadership of overall research plan, writing the first draft of the manuscript and primary responsibility for the final content of the manuscript. A B C, A D L, O A S, J S: Performing the bioinformatics and statistical analysis. A B C and A D L: Bisulfite genomic sequencing and pyrosequencing assessment. $\mathrm{M} \mathrm{S}$ : Conducting the microarray-based DNA methylation. M C C and A B C: Performing the functional and gene expression analysis. J C, R L-L, J J G, T L, O E: Sampling and clinical data collection. A D L, O A S, J S, M M-G, F J T: Interpretation of the data and critical revision of the manuscript. $\mathrm{M} E$ and $\mathrm{F} F \mathrm{C}$ acquired funding for this study. All authors: Involvement in the writing of the manuscript and approving the final version of this article. None of the authors had a conflict of interest regarding the manuscript.

Published by Bioscientifica Ltc. 


\section{Acknowledgments}

Authors thank Maria Amil, Diana García, Carles Arribas and Paula Vieiro for their technical support. We particularly wish to acknowledge the patients and the BioBank Complejo Hospitalario Universitario de Santiago (CHUS) (PT13/0010/0068) integrated in the Spanish National Biobanks Network, as well as Dr. Beatriz Fernandez and Dr Máximo Fraga for their collaboration.

\section{References}

Barrio S, Gallardo M, Albizua E, Jimenez A, Rapado I, Ayala R, Gilsanz F, Martin-Subero JI \& Martinez-Lopez J 2011 Epigenomic profiling in polycythaemia vera and essential thrombocythaemia shows low levels of aberrant DNA methylation. Journal of Clinical Pathology 64 1010-1013. (doi:10.1136/jclinpath-2011-200175)

Conway K, Edmiston SN, May R, Kuan PF, Chu H, Bryant C, Tse CK, Swift-Scanlan T, Geradts J, Troester MA, et al. 2014 DNA methylation profiling in the Carolina Breast Cancer Study defines cancer subclasses differing in clinicopathologic characteristics and survival. Breast Cancer Research 16 450. (doi:10.1186/s13058-014-0450-6)

Crujeiras AB \& Casanueva FF 2015 Obesity and the reproductive system disorders: epigenetics as a potential bridge. Human Reproduction Update 21 249-261. (doi:10.1093/humupd/dmu060)

Crujeiras AB, Cueva J, Vieito M, Curiel T, Lopez-Lopez R, Pollan M \& Casanueva FF 2011 Association of breast cancer and obesity in a homogeneous population from Spain. Journal of Endocrinological Investigation 35 681-685.

Crujeiras AB, Diaz-Lagares A, Carreira MC, Amil M \& Casanueva FF 2013 Oxidative stress associated to dysfunctional adipose tissue: a potential link between obesity, type 2 diabetes mellitus and breast cancer. Free Radical Research 47 243-256. (doi:10.3109/10715762.2013.772604)

Dick KJ, Nelson CP, Tsaprouni L, Sandling JK, Aissi D, Wahl S, Meduri E, Morange PE, Gagnon F, Grallert H, et al. 2014 DNA methylation and body-mass index: a genome-wide analysis. Lancet 383 1990-1998. (doi:10.1016/S0140-6736(13)62674-4)

Dmitriev AA, Kashuba VI, Haraldson K, Senchenko VN, Pavlova TV, Kudryavtseva AV, Anedchenko EA, Krasnov GS, Pronina IV, Loginov VI, et al. 2012 Genetic and epigenetic analysis of non-small cell lung cancer with NotI-microarrays. Epigenetics 7 502-513. (doi:10.4161/ epi.19801)

Falcon S \& Gentleman R 2007 Using GOstats to test gene lists for GO term association. Bioinformatics 23 257-258. (doi:10.1093/ bioinformatics/bt1567)

Faryna M, Konermann C, Aulmann S, Bermejo JL, Brugger M, Diederichs S, Rom J, Weichenhan D, Claus R, Rehli M, et al. 2012 Genome-wide methylation screen in low-grade breast cancer identifies novel epigenetically altered genes as potential biomarkers for tumor diagnosis. FASEB Journal 26 4937-4950. (doi:10.1096/fj.12-209502)

Hair BY, Troester MA, Edmiston SN, Parrish EA, Robinson WR, Wu MC, Olshan AF, Swift-Scanlan T \& Conway K 2015 Body mass index is associated with gene methylation in estrogen receptor-positive breast tumors. Cancer Epidemiology, Biomarkers \& Prevention 24 580-586. (doi.org/10.1158/1055-9965.epi-14-1017)

Heyn H, Li N, Ferreira HJ, Moran S, Pisano DG, Gomez A, Diez J, Sanchez-Mut JV, Setien F, Carmona FJ, et al. 2012 Distinct DNA methylomes of newborns and centenarians. PNAS 109 10522-10527. (doi:10.1073/pnas.1120658109)

Heyn H, Mendez-Gonzalez J \& Esteller M 2013 Epigenetic profiling joins personalized cancer medicine. Expert Review of Molecular Diagnostics 13 473-479. (doi:10.1586/erm.13.36)

Hill VK, Ricketts C, Bieche I, Vacher S, Gentle D, Lewis C, Maher ER \& Latif F 2011 Genome-wide DNA methylation profiling of CpG islands in breast cancer identifies novel genes associated with tumorigenicity. Cancer Research 71 2988-2999. (doi:10.1158/00085472.CAN-10-4026)
Howe LR, Subbaramaiah K, Hudis CA \& Dannenberg AJ 2013 Molecular pathways: adipose inflammation as a mediator of obesity-associated cancer. Clinical Cancer Research 19 6074-6083. (doi:10.1158/10780432.CCR-12-2603)

Li Y, Melnikov AA, Levenson V, Guerra E, Simeone P, Alberti S \& Deng Y 2015 A seven-gene CpG-island methylation panel predicts breast cancer progression. BMC Cancer 15 417. (doi:10.1186/s12885-015-1412-9)

Lorincz AM \& Sukumar S 2006 Molecular links between obesity and breast cancer. Endocrine-Related Cancer 13 279-292 (doi:10.1677/ erc.1.00729)

Luxen S, Belinsky SA \& Knaus UG 2008 Silencing of DUOX NADPH oxidases by promoter hypermethylation in lung cancer. Cancer Research 68 1037-1045. (doi:10.1158/0008-5472.CAN-07-5782)

McCullough LE, Chen J, White AJ, Xu X, Cho YH, Bradshaw PT, Eng SM, Teitelbaum SL, Terry MB, Garbowski G, et al. 2015 Gene-Specific promoter methylation status in hormone-receptor-positive breast cancer associates with postmenopausal body size and recreational physical activity. International Journal of Cancer and Clinical Research 2 013. (doi:10.23937/2378-3419/2/1/1013)

Milagro FI, Gomez-Abellan P, Campion J, Martinez JA, Ordovas JM \& Garaulet M 2012 CLOCK, PER2 and BMAL1 DNA methylation: association with obesity and metabolic syndrome characteristics and monounsaturated fat intake. Chronobiology International 29 1180-1194. (doi:10.3109/07420528.2012.719967)

Milagro FI, Mansego ML, De Miguel C \& Martinez JA 2013 Dietary factors, epigenetic modifications and obesity outcomes: progresses and perspectives. Molecular Aspects of Medicine 34 782-812. (doi:10.1016/j.mam.2012.06.010)

Naushad SM, Hussain T, Al-Attas OS, Prayaga A, Digumarti RR, Gottumukkala SR \& Kutala VK 2014 Molecular insights into the association of obesity with breast cancer risk: relevance to xenobiotic metabolism and $\mathrm{CpG}$ island methylation of tumor suppressor genes. Molecular and Cellular Biochemistry 392 273-280. (doi:10.1007/ s11010-014-2037-z)

Rauch TA, Wang Z, Wu X, Kernstine KH, Riggs AD \& Pfeifer GP 2012 DNA methylation biomarkers for lung cancer. Tumour Biology 33 287-296. (doi:10.1007/s13277-011-0282-2)

Renehan AG, Tyson M, Egger M, Heller RF \& Zwahlen M 2008 Bodymass index and incidence of cancer: a systematic review and metaanalysis of prospective observational studies. Lancet 371 569-578. (doi:10.1016/S0140-6736(08)60269-X)

Roy K, Wu Y, Meitzler JL, Juhasz A, Liu H, Jiang G, Lu J, Antony S \& Doroshow JH 2015 NADPH oxidases and cancer. Clinical Science 128 863-875. (doi:10.1042/CS20140542)

Sandoval J, Heyn H, Moran S, Serra-Musach J, Pujana MA, Bibikova M \& Esteller M 2011 Validation of a DNA methylation microarray for 450,000 CpG sites in the human genome. Epigenetics 6 692-702. (doi:10.4161/epi.6.6.16196)

Sehrawat B, Sridharan M, Ghosh S, Robson P, Cass CE, Mackey JR, Greiner R \& Damaraju S 2011 Potential novel candidate polymorphisms identified in genome-wide association study for breast cancer susceptibility. Human Genetics 130 529-537. (doi:10.1007/s00439-011-0973-1)

Seidell JC \& Halberstadt J 2015 The global burden of obesity and the challenges of prevention. Annals of Nutrition and Metabolism $\mathbf{6 6}$ Supplement 2 7-12. (doi:10.1159/000375143)

Severson PL, Tokar EJ, Vrba L, Waalkes MP, Futscher BW 2013 Coordinate H3K9 and DNA methylation silencing of ZNFs in toxicant-induced malignant transformation. Epigenetics 8 1080-1088. (doi:10.4161/epi.25926)

Stefansson OA \& Esteller M 2013 Epigenetic modifications in breast cancer and their role in personalized medicine. American Journal of Pathology 183 1052-1063. (doi:10.1016/j.ajpath.2013.04.033)

Stefansson OA, Moran S, Gomez A, Sayols S, Arribas-Jorba C, Sandoval J, Hilmarsdottir H, Olafsdottir E, Tryggvadottir L, Jonasson JG, et al. 2015 A DNA methylation-based definition of biologically distinct 
breast cancer subtypes. Molecular Oncology 9 555-568. (doi:10.1016/j. molonc.2014.10.012)

Tao MH, Marian C, Nie J, Ambrosone C, Krishnan SS, Edge SB, Trevisan M, Shields PG \& Freudenheim JL 2011 Body mass and DNA promoter methylation in breast tumors in the Western New York Exposures and Breast Cancer Study. American Journal of Clinical Nutrition 94 831-838. (doi:10.3945/ ajcn.110.009365)

Tessema M, Yingling CM, Grimes MJ, Thomas CL, Liu Y, Leng S, Joste N \& Belinsky SA 2012 Differential epigenetic regulation of TOX subfamily high mobility group box genes in lung and breast cancers. PLOS ONE 7 e34850. (doi:10.1371/journal.pone.0034850)

van Gemert WA, Lanting CI, Goldbohm RA, van den Brandt PA, Grooters HG, Kampman E, Kiemeney LA, van Leeuwen FE, Monninkhof EM, de Vries E, et al. 2015 The proportion of postmenopausal breast cancer cases in the Netherlands attributable to lifestyle-related risk factors. Breast Cancer Research and Treatment 152 155-162. (doi:10.1007/s10549-015-3447-7)

WHO 2000 Obesity:preventing and managing the global epidemic. Report of a WHO consultation. WHO technical Report Series 894. Geneva, Switzerland: World Health Organization.

Yara S, Lavoie JC \& Levy E 2013 Oxidative stress and DNA methylation regulation in the metabolic syndrome. Epigenomics 7 283-300. (doi:10.2217/epi.14.84)

Zadravec D, Brolinson A, Fisher RM, Carneheim C, Csikasz RI, BertrandMichel J, Boren J, Guillou H, Rudling M \& Jacobsson A 2010 Ablation of the very-long-chain fatty acid elongase ELOVL3 in mice leads to constrained lipid storage and resistance to diet-induced obesity. FASEB Journal 24 4366-4377. (doi:10.1096/fj.09-152298)

Zou C \& Shao J 2008 Role of adipocytokines in obesity-associated insulin resistance. Journal of Nutritional Biochemistry 19 277-286. (doi:10.1016/j.jnutbio.2007.06.006)

Received in final form 4 April 2017

Accepted 25 April 2017

Accepted Preprint published online 25 April 2017
Published by Bioscientifica Ltd. 